Nloman 2018, 36(1), 29-39

Revista de Psicologia, Ciències de l'Educació i de l'Esport

ISSN: $1138-3194$

Copyright (c) 2018

www.revistaaloma.net

\title{
Uso del móvil en padres, niños y adolescentes: Creencias acerca de sus riesgos y beneficios
}

\author{
Gloria Besoli, Núria Palomas \& Andrés Chamarro \\ Universidad Autónoma de Barcelona
}

Recibido: 29-1-2018

Aceptado: 3-4-2018

Uso del móvil en padres, niños y adolescentes: Creencias acerca de sus riesgos y beneficios

Resumen. Se observa una creciente penetración del móvil entre los menores, que indudablemente se hace de la mano de sus padres. Pese a que el móvil ofrece oportunidades de comunicación y socialización, la literatura científica y los medios de comunicación ponen énfasis en los riesgos que su uso supone para los menores y adolescentes. Por ello, la presente investigación estudia los usos, riesgos y beneficios del teléfono móvil, sobre todo en menores, y analiza las creencias de los padres acerca de esta tecnología. Adicionalmente analiza la relación entre el uso problemático del móvil de los menores y su satisfacción vital. Se realizan dos estudios, uno con 63 padres y otro con 411 estudiantes de primaria, secundaria y bachillerato. Los resultados indican que los usos principales son comunicativos, recreativos y prácticos, sugieren que en el momento actual el uso práctico y con potenciales beneficios parece destacar. También se ha encontrado que el tiempo y la frecuencia de uso por parte de los adultos y los menores parecen equipararse, lo que sugiere un incremento progresivo del uso en los adolescentes. Se observa que el uso problemático del móvil es mayor en secundaria y bachillerato que en primaria, con un $15 \%$ de los adolescentes en situación de riesgo de uso problemático. Se concluye que los menores están evolucionando del uso principalmente lúdico al uso comunicativo, facilitado por la eclosión de las redes sociales, lo que indica que integran la tecnología en sus hábitos de vida y la utilizan de una forma positiva. Estos resultados contrastan con la percepción de los padres respecto de los usos del móvil por parte de los hijos, que se limita al uso lúdico y obvian el uso comunicativo potenciado por las redes sociales.

Palabras clave: Teléfono móvil; smartphone; uso problemático del móvil; satisfacción vital; redes sociales

Mobile phone use in parents, children and adolescents: Beliefs about risks and benefits

Summary. Mobile phone use has become ever more prevalent among minors, doubtless thanks to the support of their parents. While it is true that mobiles offer great opportunities for communication and socialising, the scientific literature and the media often focus on the risks that mobile use can pose to children and adolescents. With this in mind, this paper examines the uses, risks and benefits of mobile phone use, specifically with regard to minors, and it analyses parents' beliefs connected with this technology. It goes on to analyse the connection between problematic mobile use in minors and their degree of satisfaction with their lives. Two studies were conducted, one of 63 parents and the other of 411 primary and high school students. The results indicate that the most frequent uses of these devices are for communication, recreation and practical purposes. The results also suggest that these practical uses and the beneficial effects are currently most prominent. The findings also indicate that the time spent using the devices and the frequency of use are similar in adults and children, which suggests a gradual increase in use among adolescents. Problematic mobile phone use is more prevalent in high school than in primary school, with $15 \%$ at risk of problematic use. The study concludes that children are moving from primarily using the devices for leisure to greater communicative use, thanks in part to the expansion of social networks. This indicates that they are incorporating technology into their life habits and making positive use of it. This contrasts with parents' perceptions of their children's mobile use as being limited to play and leisure. Parents do not seem to realise the communicative potential of social networks on mobiles for children and adolescents.

Keywords: mobile phones; smartphones; problematic mobile phone use; life satisfaction; social networks

Correspondence

Andrés Chamarro

http://orcid.org/0000-0002-8059-5467

Universitat Autònoma de Barcelona

andres.chamarro@uab.es 


\section{Introducción}

El teléfono móvil se ha convertido, en los últimos años, en un elemento imprescindible en la vida de nuestros adolescentes y ha transformado, además, la forma de relacionarse (Ruiz, Sánchez \& Trujillo, 2016). Su rápido crecimiento se observa en las altas tasas de penetración. La Encuesta sobre Equipamiento y Uso de Tecnologías de la Información y la Comunicación en los Hogares (Instituto Nacional de Estadística, 2016) muestra que el 99.3\% de los hogares españoles disponen de algún tipo de teléfono para comunicarse y que el $96.3 \%$ lo hace mediante teléfono móvil. No solo ha entrado con fuerza en los hogares españoles, sino que el número de teléfonos móviles que hoy están en funcionamiento es similar al número de habitantes. El Informe Mobile en España y en el Mundo, elaborado por Ditrendia (2016), señala que el 89\% de los españoles mayores de 13 años posee un teléfono móvil y, de entre el total de estos dispositivos, el $87 \%$ es un smartphone.

Desde 2010 se observa una rápida expansión del uso del móvil, especialmente en las edades más bajas. Así lo confirma el estudio elaborado por Bringué y Sábada (2011), que sitúa la edad de inicio a partir de los 8 años (55\% se inician entre los 8 y 11 años). El móvil se suma a otras pantallas presentes en las vidas de los menores. Esta proliferación de dispositivos, especialmente los móviles, está propiciando la adopción de nuevas pautas de conducta y afectando a sus relaciones personales y familiares (Martínez de Morentín, Cortés, Medrano \& Apodaca, 2014). Muñoz-Miralles et al. (2016) señalan el aumento de la vulnerabilidad al desarrollo de adicciones, tanto de carácter comportamental como de consumo de sustancias tóxicas, además de la disminución del contacto presencial en favor de la interacción virtual.

En general, los autores destacan las potenciales consecuencias negativas, tanto físicas como psicológicas y sociales, derivadas del uso del móvil. Entre ellas encontramos problemas médicos, riesgos para la seguridad vial, la intimidación y el acoso. A ello habría que añadir los comportamientos derivados de la mala adaptación, como por ejemplo el uso excesivo, que pueden afectar a los sentimientos de seguridad personal, a la identidad y a la pertenencia a grupos sociales (Carbonell, Oberst \& Beranuy, 2013). La incapacidad para desprenderse del móvil, la constante comprobación del estado de la batería o cobertura, así como la tendencia a sentirse receptores de mensajes o llamadas constantemente, podrían estar reflejando pautas desadaptativas de uso a pesar de que ni jóvenes ni mayores las identifiquen con un posible uso problemático del teléfono móvil (Instituto Nacional de Tecnologías de la Comunicación, 2010). Son aspectos como la relación con los demás (Rial, Gómez, Varela \& Braña, 2014), el bajo rendimiento académico y las alteraciones del sueño (Muñoz-Miralles et al., 2014), o cuestiones como el prestigio y la identidad (McBride, 2011; Ruiz et al., 2016), las que preocupan a los usua- rios, pero lo que al parecer es lo más alarmante es la exposición al acoso, sobre todo desde la aparición de las plataformas de mensajería instantánea (Fundación ANAR, 2016). Algunos adolescentes ven en las redes sociales la principal forma de interacción social, y su uso conlleva que gran parte de su vida social y su desarrollo emocional ocurra en ese nuevo espacio de relación. No podemos olvidar que su capacidad de autorregulación es limitada y que son más vulnerables a la presión del grupo, por lo que están en riesgo mientras navegan y experimentan con las redes sociales (McBride, 2011). Esto supone que los padres deberán afrontar nuevos riesgos inherentes a la socialización de sus hijos (Romero-Ruiz et al., 2016).

Pese a la gran cantidad de riesgos identificados, el aspecto más controvertido y que genera más alarma social sigue siendo su posible potencial adictivo (Labrador \& Villadangos, 2010; Muñoz-Miralles et al., 2016; Salehan \& Negahban, 2013), a pesar de que afirmar si existe o no la adicción al móvil no parece una tarea sencilla, puesto que como ponen de relieve Carbonell y Panova (2017), que el uso del móvil tenga consecuencias negativas no lo convierte en adicción. Tras el dilatado debate que diversos autores han llevado a cabo, de la literatura científica se desprende la tendencia a hablar de uso desadaptativo como término más acertado, y parece, además, que este tipo de uso podría remitir en adultos y adolescentes con las adecuadas pautas educativas (Carbonell, Fuster, Chamarro \& Oberst, 2012).

En todo caso, más allá del uso problemático algunos autores han planteado la existencia de beneficios asociados al uso del móvil. El desarrollo de nuevos patrones de uso para actividades rutinarias (p. ej., creatividad) (O'Keeffe, Clarke-Pearson, \& Council on Communications and Media, 2011) o el mantenimiento de las relaciones familiares (Carvalho, Fonseca, Francisco, Bacigalupe, \& Reivas, 2016) y sociales (Malo, Navarro, \& Casas, 2012) se destacan como aspectos positivos del uso del teléfono móvil. Asimismo, la actitud positiva hacia uno mismo que deriva de las relaciones con amigos (Rial et al., 2014) y la facilidad de comunicación e incluso el uso del móvil como dispositivo para combatir el aburrimiento (Beranuy, Chamarro, Graner, \& Carbonell, 2009) pueden ser ejemplos de otras consecuencias positivas.

Si esto es así, el uso del móvil por parte de los menores puede tener dos tipos de efecto: uno negativo, derivado de la experimentación de sentimientos de soledad y abandono de las relaciones y otro positivo, ya que la comunicación en línea estimula el bienestar a través del tiempo invertido con los amigos y la calidad de estas amistades (Valkenburg \& Peter, 2009). Valkenburg, Peter y Schouten (2006) señalan que cuanto más se usan las redes sociales mayor es la interacción por lo que aumenta la autoestima y, en última instancia, la satisfacción con la vida, especialmente en la adolescencia.

A pesar de la variedad de interrogantes que se abren en torno a las consecuencias del uso de la tecnología 
móvil, actualmente tanto la literatura científica como la opinión pública insisten en destacar los peligros, lo que pone de manifiesto que las preocupaciones en relación con el uso del móvil persisten. Sin embargo, las familias parecen no ser conscientes de la influencia que puede tener el hábito del uso del móvil, y el acceso a las redes sociales que posibilita en sus hijos (Valencia, Sánchez, Garcés \& González, 2015), por lo que, como señalan Gallimberti et al. (2016), el uso del móvil puede ser visto como un potencial problema de salud pública que puede dar lugar a problemas sociales, conductuales y afectivos. Para Plaza de la Hoz (2017) las familias tienen un papel clave en el desarrollo de un uso saludable de la tecnología por parte de los menores y los padres deben mejorar sus habilidades de enseñanza para poder empoderar a sus hijos con autonomía y autocontrol. Pese a ello, las investigaciones revelan falta de capacitación en los adultos responsables de los niños de menor edad tal vez relacionada con la baja percepción de los riesgos.

Dada la relevancia del problema y el escaso conocimiento existente, en este estudio pretendemos explorar los riesgos y beneficios del uso del teléfono móvil, sobre todo en menores. Considerando que la expansión del uso de los teléfonos móviles en los menores viene de la mano de sus padres, es necesario conocer sus actitudes acerca de esta tecnología, dado que el uso que los adultos hacen del móvil puede estar condicionando el uso que hacen los menores y que la percepción que los mayores tienen acerca de los riesgos y beneficios puede no coincidir con lo que los menores destacan como experiencias significativas.

Para cumplir con este objetivo se realizaron dos estudios: El estudio 1 centrado en los usos que hacen los adultos del móvil y su percepción de los riesgos y beneficios del uso del móvil por parte de sus hijos. El estudio 2 centrado en conocer el uso que hacen los adolescentes, su percepción sobre los riesgos y beneficios, y su relación con consecuencias negativas percibidas y su satisfacción con la vida.

\section{Estudio 1}

\section{Método}

\section{Participantes}

Participaron 63 padres y madres con hijos comprendidos entre los 10 y los 12 años que tuvieran teléfono móvil o acceso a él. Cada familia participante seleccionó un sujeto adulto de opinión (padre, madre o tutor legal). Los datos se recogieron previo consentimiento informado. De los 63 encuestados, 43 fueron mujeres (68\%) y 20 hombres (32\%). La edad media fue de 45,32 años (DT = 3.41), con un rango de edad entre los $35 \mathrm{y}$ los 52 años. El nivel educativo más frecuente eran los estudios universitarios (63\%), seguidos de FP o bachillerato $(30 \%)$.

\section{Instrumentos}

Se solicitó información sociodemográfica (edad, sexo, tipología familiar y nivel académico).

Basándonos en el estudio de Pontes, Szabo y Griffiths (2015), se solicitaron datos relacionados con la frecuencia de uso y el tiempo de uso del dispositivo móvil, tanto del adulto como de lo que este piensa que hace el menor, y de las funciones más destacadas del dispositivo. Para la frecuencia de uso se contemplaron consultas cada 30 minutos o menos, entre 30 y 60 minutos, cada hora, cada 3 horas y cada 6 horas. Para el tiempo de consulta se contempló un total de 30 minutos o menos, de 30 a 60 minutos, de 1 a 3 horas, de 3 a 6 horas y más de 6 horas. De los diferentes usos que se pueden dar al teléfono móvil se preguntó a los participantes por los que se consideraron más relevantes (voz, videoconferencia, chat, redes sociales, web, compras, juegos, contenidos multimedia, cámara de fotos, descarga de aplicaciones, reproductor de audio y tareas administrativas). Las apuestas y la navegación por páginas de contenido para adultos no fueron incluidas en este estudio por no considerarse propias del uso de los menores. La escala de respuesta era tipo Likert de 5 puntos, con opciones de respuesta comprendidas entre 0 (nunca) y 4 (siempre).

Para las creencias se optó por elaborar un cuestionario adhoc con ítems relacionados con algunas de las consecuencias de uso, tanto positivas como negativas (p. ej., Beranuy et al., 2009). Se elaboraron dos conjuntos de ítems para recopilar, por una parte, lo que piensan los adultos sobre el uso del teléfono móvil en general y, por otra, lo que piensan en relación con el uso que los menores pueden hacer del teléfono móvil.

Los 33 ítems del primer bloque medían cuestiones relacionadas con la seguridad y el control, relaciones interpersonales, riesgos para la salud (física y psicológica), gratificaciones, desarrollo personal e intimidad. Se sometieron a análisis factorial exploratorio y se desestimaron 21. Los 12 restantes quedaron agrupados en 3 categorías: (a) beneficios percibidos, que comprende ítems como el aumento de la sensación de tranquilidad para uno mismo, la estimulación de la comunicación o la mejora de la vida (p. ej., El uso del móvil hace más fácil que hable con otras personas); (b) inconvenientes percibidos, que incluye ítems relativos a la posibilidad de provocar accidentes de circulación, problemas de visión o la modificación del estilo comunicativo (p. ej., El uso del móvil hace que la manera de explicar las cosas sea diferente a la que estamos acostumbrados), y (c) dificultad de control, que contiene ítems como considerarse un adicto al móvil, el uso durante las comidas o durante el descanso nocturno (p. ej., Creo que estoy demasiado pendiente del móvil). Posteriormente, esta estructura de tres factores se sometió a análisis factorial confirmatorio. La solución mostraba un ajuste aceptable a los datos $\left(\chi^{2}(66)=924.17 ; \mathrm{p}<0.001 ; \mathrm{GFI}=.94\right.$; RMSEA $=.069$ [.056 - .082]).

De los 55 ítems del segundo bloque, mediante análisis factorial exploratorio se desestimaron 26 y el resto 
se agruparon en tres dimensiones: (a) crecimiento personal, que comprende ítems acerca de la facilitación de los vínculos intergeneracionales, el desarrollo de la responsabilidad del menor y la ampliación del número de amistades (p. ej., El teléfono móvil en menores facilita el contacto con los padres, abuelos y primos); (b) efectos adversos, que comprende ítems relativos a entorpecer la capacidad de planificar o provocar dependencia (p. ej., El teléfono móvil en menores interfiere en las tareas escolares), y (c) efectos adversos, que comprende ítems como aumento de la posibilidad de exponerse al grooming, al sexting, al ciberbullying o que favorece el desarrollo de conductas impulsivas (p. ej., El teléfono móvil en menores facilita el acoso por parte de compañeros). Esta estructura de tres factores se sometió a análisis factorial confirmatorio. La solución mostraba un ajuste aceptable a los datos $\left(\chi^{2}(325)=2878.25 ; \mathrm{p}<0.001 ; \mathrm{GFI}=.94\right.$; RMSEA $=.086$ [.057-.088]).

Todas las puntuaciones se registraron con base en una escala tipo Likert de 5 opciones oscilante entre 1 (totalmente de acuerdo) y 5 (totalmente en desacuerdo).

\section{Procedimiento}

El cuestionario se distribuyó a través de grupos de WhatsApp de familias de $5 .^{\circ}$ y $6 .^{\circ}$ de primaria, en Barcelona. Se proporcionó información sobre el objetivo del cuestionario y se solicitó el consentimiento informado. Asimismo, se facilitaron instrucciones para garantizar la correcta cumplimentación del mismo. Los datos se recogieron desde mediados de julio hasta finales de octubre de 2016.

\section{Análisis de datos}

Se obtuvieron estadísticos descriptivos de las variables de estudio. También se calcularon las correlaciones para las variables de uso del móvil y creencias. El análisis de los datos se realizó con el paquete estadístico SPSS 17 y la hoja de cálculo Microsoft Excel versión 14.5.5.

\section{Resultados}

\section{Uso del teléfono móvil}

Los estadísticos descriptivos para el uso del móvil pueden ser consultados en la Tabla 1. En lo relativo al tiempo de uso del teléfono móvil por parte de los padres, el uso mayoritario (51\%) es de entre 30 y 60 minutos. Un 30\% adicional lo utiliza de 1 a 3 horas diarias. Respecto a la frecuencia de consulta, informan consultar su teléfono móvil cada 30 minutos o menos (32\%) o cada hora (40\%). Respecto a los menores, el 65\% de los adultos creen que usan el móvil un tiempo inferior a 30 minutos diarios y con una frecuencia de consulta de 1 vez al día (51\% de los encuestados).

De los diferentes usos presentados el más frecuente es el de mensajería instantánea, el 97\% asegura usarlo siempre o casi siempre. También aparecen como uso habitual las consultas web (68\%), utilizar la cámara de fotos $(68 \%)$ y emitir o recibir llamadas de voz (51\%). El uso menos frecuente es la videoconferencia, un 92\% dice no usarlo nunca o rara vez. El 90\% de los encuestados dice usar poco el teléfono móvil para acceder a contenidos multimedia. El 89\% refiere no usar los juegos frecuentemente. El 86\% dice no hacer compras en línea y un $73 \%$ asegura realizar escasamente tareas administrativas. El 65\% de los adultos refiere no hacer uso ni de las descargas de aplicaciones ni de la reproducción de música desde el dispositivo.

Por lo que se refiere al uso que los adultos creen que los menores hacen del teléfono móvil, cabe destacar que más del 90\% piensa que el menor no usa el móvil para realizar llamadas de voz, ni para realizar tareas administrativas, comprar o participar en videoconferencias. Los usos estimados que aparecen como los más frecuentes en los menores son los juegos (51\% para las frecuencias siempre y casi siempre), la cámara de fotos (un 35\%), la mensajería instantánea (33\%) y escuchar música (30\%).

\section{Creencias de los adultos en relación con el uso del smartphone en general}

Los estadísticos descriptivos para las creencias aparecen en la Tabla 1. De acuerdo con la escala utilizada, los beneficios se perciben moderados y los inconvenientes se perciben bajos. Los indicadores de dependencia son percibidos como moderadamente bajos. No se observan diferencias estadísticas entre padres y madres.

\section{Creencias de los adultos en relación con el uso que hacen los menores del smartphone}

Las creencias paterna y materna relacionadas con el crecimiento personal, los efectos adversos y los riesgos para el menor, son de magnitud moderada. En cuanto a diferencias de género, no se aprecian para ninguna de estas categorías.

\section{Correlaciones}

Respecto a los usos, las llamadas de voz se correlacionan moderadamente con la mensajería instantánea, y la mensajería con las consultas web y el uso de la cámara fotográfica. También presentan correlación moderada las descargas con escuchar música, que a su vez presenta una correlación moderada e inversa con las tareas administrativas. Visualizar contenidos multimedia se correlaciona moderadamente con la descarga de contenidos y en menor medida con la realización de tareas administrativas. Usar el smartphone para jugar presenta correlación moderada con las descargas, con el visionado de contenidos multimedia y en menor medida con escuchar música. Cabe destacar también la correlación moderada que presentan los usos de consulta web con las compras en línea. Respecto al tiempo de dedicación se observa una correlación moderada negativa con la frecuencia de uso. 
Tabla 1. Estadísticos descriptivos y correlaciones para las variables de uso del móvil y las creencias en los padres

\begin{tabular}{|c|c|c|c|c|c|c|c|c|c|c|c|c|c|c|c|c|c|c|c|c|c|c|c|c|}
\hline & Media & SD & $\alpha$ & Rango & 1 & 2 & 3 & 4 & 5 & 6 & 7 & 8 & 9 & 10 & 11 & 12 & 13 & 14 & 15 & 16 & 17 & 18 & 19 & 20 \\
\hline 1 Uso: Llamada de voz & 2.49 & 1.24 & & $1-5$ & - & .24 & $.37^{\star \star}$ & .01 & .15 & .05 & -.09 & -.09 & .18 & -.19 & -.11 & -.13 & .01 & -.13 & .05 & .16 & -.10 & -.09 & .17 & .13 \\
\hline 2. Uso: Videoconferencia & 2.90 & 1.13 & & $1-5$ & & - & .13 & -.08 & .13 & $.29^{*}$ & -.08 & .14 & .23 & .01 & .10 & .15 & .02 & -.07 & -.05 & .20 & -.07 & -.20 & .07 & .13 \\
\hline $\begin{array}{l}\text { 3. Uso: Mensajería } \\
\text { instantánea }\end{array}$ & 3.29 & 1.10 & & $1-5$ & & & - & $.27^{\star}$ & $.47^{\star \star}$ & .15 & .07 & $.27^{*}$ & $.44^{\star \star}$ & ,05 & .04 & .17 & -.02 & -.08 & .02 & -.15 & -.02 & .00 & -.03 & -.03 \\
\hline 4 Uso: Redes sociales & 2.90 & 1.13 & & $1-5$ & & & & - & .20 & .05 & .11 & .11 & $.26^{*}$ & -.01 & -.04 & .17 & .01 & .20 & .00 & -.03 & .03 & .00 & .03 & -.01 \\
\hline 5. Uso: Consulta web & 2.75 & 0.80 & & $1-5$ & & & & & - & $.44^{\star *}$ & $.26^{*}$ & .16 & $.31^{*}$ & .24 & .19 & .21 & -.04 & -.17 & -.02 & .09 & -.19 & .06 & .07 & .03 \\
\hline 6. Uso: Compras & 3.03 & 0.77 & & $1-5$ & & & & & & - & $.32^{\star *}$ & .13 & .16 & $.35^{\star *}$ & $.41^{\star *}$ & $.37^{\star \star}$ & -.20 & -.16 & -.05 & -.08 & -.12 & .03 & -.02 & .05 \\
\hline 7. Uso: Juegos & 2.11 & 1.23 & & $1-5$ & & & & & & & - & $.40^{\star *}$ & .07 & $.42^{* *}$ & $.37^{* \star}$ & $.25^{\star}$ & .06 & -.16 & .16 & -.10 & .02 & .10 & -.07 & -.09 \\
\hline 8. Uso: Películas & 2.49 & 1.11 & & $1-5$ & & & & & & & & - & $.30^{*}$ & $.46^{\star \star}$ & $.32^{*}$ & $.32^{\star \star}$ & $-.25^{*}$ & .02 & .03 & -.13 & -.03 & -.12 & .12 & -.15 \\
\hline 9. Uso: Fotografía & 2.19 & 1.25 & & $1-5$ & & & & & & & & & - & $.28^{*}$ & $.27^{*}$ & .22 & .02 & -.10 & -.05 & .00 & -.11 & -.08 & .13 & .07 \\
\hline 10.Uso: Descargas & 2.06 & 1.06 & & $1-5$ & & & & & & & & & & - & $.49^{\star \star}$ & $.35^{\star \star}$ & .00 & $-.29^{*}$ & -.05 & -.05 & $-.26^{*}$ & -.02 & -.04 & -.04 \\
\hline 11. Uso: Música & 2.26 & 1.40 & & $1-5$ & & & & & & & & & & & - & $-46^{* *}$ & -.13 & -.03 & .06 & .08 & -.24 & -.15 & .03 & .07 \\
\hline 12. Uso: Administración & 3.41 & 1.20 & & $1-5$ & & & & & & & & & & & & - & -.13 & .12 & -.10 & .05 & -.05 & -.19 & -.16 & .01 \\
\hline 13. Frecuencia & 3.63 & 1.23 & & $1-5$ & & & & & & & & & & & & & - & $-.45^{\star \star}$ & .00 & .00 & -.07 & .08 & .07 & .13 \\
\hline 14. Tiempo & 3.78 & 1.33 & & $1-5$ & & & & & & & & & & & & & & - & .05 & .10 & .03 & -.09 & -.05 & -.01 \\
\hline 15. Beneficios & 9,10 & 2.30 & .43 & 3-15 & & & & & & & & & & & & & & & - & -.06 & $.29^{*}$ & $.52^{\star *}$ & .20 & .16 \\
\hline 16. Riesgos & 6.79 & 3.12 & .83 & $3-15$ & & & & & & & & & & & & & & & & - & $-.35^{* *}$ & $-.36^{* *}$ & $.45^{\star *}$ & $.53^{\star \star}$ \\
\hline 17. Dificultad de control & 20.84 & 5.09 & .76 & 9-30 & & & & & & & & & & & & & & & & & - & $.29^{*}$ & .10 & -.07 \\
\hline 18. Crecimiento personal & 14.88 & 3.84 & .70 & $5-25$ & & & & & & & & & & & & & & & & & & - & -.02 & -.06 \\
\hline 19. Inconvenientes & 24.61 & 7.82 & .88 & $10-47$ & & & & & & & & & & & & & & & & & & & - & $.82^{\star *}$ \\
\hline 20. Efectos adversos & 17.65 & 7.13 & .91 & $8-40$ & & & & & & & & & & & & & & & & & & & & - \\
\hline
\end{tabular}

Nota. ${ }^{*} \mathrm{p}<.05,{ }^{* *} \mathrm{p}<.001$

Para las creencias, los beneficios percibidos por los adultos y el crecimiento personal para los menores se observa una correlación moderada. Asimismo, los inconvenientes percibidos por los adultos se correlacionan moderadamente con los riesgos para los menores y los efectos adversos. Efectos adversos y riesgos muestra una correlación muy elevada. Finalmente cabe señalar que los inconvenientes percibidos en el uso del móvil por parte de los padres muestran una correlación moderada con la dependencia y una correlación negativa con el crecimiento personal para los menores.

\section{Discusión}

Este estudio tenía por objetivo conocer los usos que hacen los adultos del móvil y su percepción de los riesgos y beneficios del uso del móvil por parte de sus hijos. Los resultados de los diferentes usos sugieren que estos se pueden agrupar en: (a) usos comunicativos, que englobarían llamadas de voz, videoconferencia, mensajería instantánea, fotografía y redes sociales; (b) usos recreativos, que englobarían los juegos, la visualización de contenidos multimedia, las descargas y la música, y (c) usos prácticos, que contendrían las consultas web, las compras y las tareas administrativas. El uso comunicativo va asociado a las consultas web y el uso de la cámara fotográfica, por lo que el tipo de comunicación se hace cada vez más gráfica y menos textual. Estos usos son parcialmente coincidentes con los identificados por Pontes et al. (2015), que encontraron que los usos más frecuentes eran navegar por Internet, participar en las redes sociales y comunicarse mediante correo o chat. La diferencia puede explicarse por el hecho de que la muestra del estudio aquí expuesto se centra en el uso del móvil como dispositivo, por lo que las llamadas de voz siguen siendo una actividad frecuente.
La mayoría de los progenitores declaran hacer un uso más comunicativo que ocioso, en contraste con el uso que creen que hacen los menores. Esto es consistente con lo que señalan Carbonell et al. (2013) sobre el uso de los móviles por parte de menores, más orientado al juego que a la comunicación, ya que todavía es demasiado abstracta para ellos. También podría responder a lo que Moral y Suárez (2016) consideran la normalización del uso del móvil: a medida que aumenta la edad su uso se vuelve más pragmático y menos lúdico. El uso comunicativo del móvil mediante acceso a redes sociales parece destacado tanto para adultos como para menores, en línea con los hallazgos de estudios previos (Salehan \& Negahban, 2013). Respecto a los tiempos y las frecuencias de uso, la correlación negativa parece indicar que cuanto más tiempo se usa el teléfono menos frecuentemente se consulta, lo cual resulta comprensible pues la consulta se vuelve innecesaria.

Respecto a las creencias paternas y maternas, el móvil parece ser visto como un dispositivo cuyo uso es beneficioso (Carvalho et al., 2016; Rial et al., 2014), pero que al mismo tiempo conlleva múltiples riesgos e inconvenientes (Gallimberti et al., 2016). Esta tendencia a señalar aspectos negativos es inconsistente con el uso real del móvil, ya que los datos relativos a los usos recogidos en este estudio no se correlacionan con los riesgos percibidos.

En definitiva, los datos revelan que los adultos de esta muestra son conscientes de las utilidades del teléfono móvil, pero también de las implicaciones de su uso problemático y consideran que valoran bien los riesgos para los menores. Como señalan Sánchez-Carbonell, Beranuy, Castellana, Chamarro, y Oberst (2008), los jóvenes, y especialmente los infantes, son el grupo que presenta mayor riesgo porque aún no tienen el control sobre sus impulsos y son fácilmente influenciables por el entorno y los demás. 


\section{Estudio 2}

Este estudio tiene el objetivo de: a) conocer los usos del teléfono móvil de los adolescentes; b) conocer qué creencias muestran respecto a él, y c) relacionar los usos, con las creencias, el uso problemático y el bienestar.

\section{Participantes}

Participaron 411 estudiantes desde $5 .^{\circ}$ de primaria hasta $2 .^{\circ}$ de bachillerato. El rango de edad fue de 9 a 18 años con una media de 13.29 años ( $\mathrm{SD}=2.27$ ). En cuanto al género, 210 (51.1\%) eran chicos y 201 (48.9\%) chicas. Un $26.5 \%$ cursaban estudios de primaria, un $65.7 \%$ de secundaria y un $7.2 \%$ de bachillerato. El $85.2 \%$ poseía un teléfono móvil propio. Se obtuvieron respuestas completas para todos los sujetos, por lo que no hubo que desestimar ningún caso posteriormente.

\section{Instrumentos}

La recogida de datos se realizó mediante tres cuestionarios:

El cuestionario de creencias relacionadas con lo que piensan los mayores en relación con el uso que los menores pueden hacer del teléfono móvil, utilizado en el estudio 1. Recogía datos relativos al uso del móvil: llamadas de voz, videoconferencias, mensajería instantánea, redes sociales, consulta de webs, compras en línea, juegos, ver películas, fotografía, descargas, escuchar música, administración, y duración y frecuencia del uso.

El cuestionario de experiencias relacionadas con el móvil (CERM) desarrollado por Beranuy et al. (2009) que mide el uso problemático del móvil. Este cuestionario comprende 10 ítems tipo Likert con 4 respuestas por orden de intensidad creciente. Está compuesto de 2 factores: conflictos y uso comunicativo/emocional. La fiabilidad obtenida en el presente estudio es adecuada (alfa de Cronbach $=.85$ ).

La Escala de Satisfacción con la Vida desarrollada por Diener, Emmons, Larsen, y Griffin (1985). Se utilizó la traducción al castellano de Atienza, Pons, Balaguer, y García-Merita (2000). Consta de 5 ítems que evalúan la satisfacción con la vida a través del juicio global que hacen las personas sobre esta. Es una escala de un solo factor que ha mostrado una alta consistencia interna con valores de alfa de Cronbach que oscilan entre .89 y .79 (p. ej., Padrós, Gutiérrez, \& Medina, 2015). La fiabilidad en el presente estudio es de .86.

\section{Procedimiento}

Los datos se recogieron durante el mes de octubre de 2016 en las aulas de un centro educativo concertado de Sabadell. Se administraron los mismos cuestionarios a cada curso. Los alumnos de primaria respondieron los cuestionarios en papel, facilitados y supervisados por el investigador. Los alumnos de secundaria y bachillerato accedieron a los cuestionarios a través de un enlace en línea utilizando ordenadores o sus propios móviles. La primera parte de los cuestionarios presentaba los objetivos del estudio y señalaba el carácter confidencial y anónimo de las respuestas. Durante la recogida de datos, el investigador estuvo en cada clase explicando en qué consistía el estudio y resolviendo dudas o problemas con los ítems en aquellos alumnos que lo necesitaron. La participación de los estudiantes fue autorizada por la dirección del centro.

\section{Análisis de datos}

El análisis de datos se realizó con el programa estadístico SPSS versión 23.0 en español. Las técnicas usadas fueron: 1) Análisis descriptivo; 2) análisis de correlaciones, y 3) análisis de varianza para las creencias acerca de los riesgos y beneficios por parte de los padres del estudio 1 y los alumnos de la muestra 2, y también en función del ciclo de estudios (primaria, secundaria y bachillerato).

\section{Resultados}

\section{Uso del teléfono móvil}

En lo relativo al tiempo de uso del teléfono móvil por parte de los adolescentes, la mayoría (37\%) lo utiliza de 1 a 3 horas diarias. Un 30\% adicional entre 30 y 60 minutos, y un $20.8 \%$ de 0 a 30 minutos. Respecto a la frecuencia de consulta, la principal es de cada $30 \mathrm{mi}-$ nutos o menos $(28.8 \%)$ o cada hora $(25.2 \%)$.

De los diferentes usos presentados, los más frecuentes son los de mensajería instantánea y redes sociales, el $74.6 \%$ y $60.8 \%$ aseguran usarlos siempre o casi siempre. También aparecen como uso habitual la cámara de fotos (57.4\%), escuchar música (59.7\%), jugar a videojuegos (40.2\%), ver películas y videos (39.5\%), descargar aplicaciones (39.3\%), las consultas web (34.6\%) y, en menor medida, emitir o recibir llamadas de voz (31\%). Entre los usos menos frecuentes encontramos la videoconferencia, un $78.7 \%$ dicen no usarla nunca o rara vez, las compras en línea (90.1\%) y las tareas administrativas (97.1\%).

\section{Correlaciones}

En la Tabla 2 se pueden consultar las correlaciones entre las variables estudiadas. El uso problemático del móvil y el bienestar se correlaciona negativamente. El uso problemático del móvil se correlaciona positivamente con la mensajería instantánea, el uso de redes sociales, el hecho de escuchar música, la frecuencia de uso, y, moderadamente, con el tiempo de uso y los inconvenientes percibidos. Se correlaciona negativamente con los beneficios, la dificultad de control y las ventajas. Los efectos adversos tienen una correlación positiva con riesgos e inconvenientes. Las ventajas se correlacionan, moderada y positivamente, con los beneficios y el con- 
Tabla 2. Estadísticos descriptivos y correlaciones para las variables de uso del móvil, riesgos y beneficios, en menores y adolescentes

\begin{tabular}{|c|c|c|c|c|c|c|c|c|c|c|c|c|c|c|c|c|c|c|c|c|c|c|c|}
\hline & Media & DT & 1 & 2 & 3 & 4 & 5 & 6 & 7 & 8 & 9 & 10 & 11 & 12 & 13 & 14 & 15 & 16 & 18 & 19 & 20 & 21 & 22 \\
\hline 1 Llamada de voz & 2.91 & 1.16 & - & $.27^{\star *}$ & $.48^{* *}$ & $.37^{* *}$ & $.33^{\star \star}$ & $.20^{\star *}$ & .073 & $.25^{\star *}$ & $.46^{\star *}$ & $.43^{\star *}$ & $.37^{\star *}$ & .05 & $.29^{* *}$ & $.40^{\star *}$ & $-.29^{* *}$ & .059 & $-.24^{\star *}-.21^{\star *}$ & .021 & .010 & $.249^{* *}$ & -.073 \\
\hline 2. Videoconferencia & 1.68 & .99 & & - & $.15^{\star *}$ & $.18^{\star *}$ & $.25^{* \star}$ & $.31^{\star *}$ & $.12^{\star *}$ & $.22^{\star \star}$ & $.31^{\star *}$ & $.34^{\star *}$ & $.31^{\star *}$ & $.21^{\star *}$ & $.16^{* *}$ & $.19^{* *}$ & $-.20^{\star \star}$ & -.033 & $-.22^{* *}-.19^{* *}$ & -.08 & -.01 & $.17^{* *}$ & -.04 \\
\hline 3. Mensajería instantánea & 4.07 & 1.34 & & & - & $.53^{\star \star}$ & $.39 * *$ & $.16^{\star \star}$ & .01 & $.27^{\star *}$ & $.43^{\star \star}$ & $.37^{\star \star}$ & $.45^{\star \star}$ & .075 & $.50^{\star *}$ & $.52^{\star \star}$ & $-.39^{* \star}$ & .064 & $-37^{\star *}-.29 * *$ & -.00 & -.04 & $.38^{* *}$ & $-.10^{*}$ \\
\hline 4. Redes sociales & 3.49 & 1.45 & & & & - & $.38^{* *}$ & $.23^{\star *}$ & -.005 & $.22^{\star \star}$ & $.42^{\star \star}$ & $.35^{\star \star}$ & $.50^{\star \star}$ & .095 & $.47^{\star *}$ & $.46^{* *}$ & $-.26^{\star \star}$ & .055 & $.-33^{* \star}-.23^{\star *}$ &.-05 & -.05 & $.32^{\star *}$ & -.07 \\
\hline 5. Consulta web & 3.10 & 1.08 & & & . & & & $.32^{\star *}$ & .067 & $.36^{\star \star}$ & $.36^{\star \star}$ & $.39^{* *}$ & $.38^{* \star}$ & $.114^{*}$ & $.30^{\star *}$ & $.32^{* *}$ & $-.25^{\star \star}$ & -.003 & $-.23^{* *}-.21^{* *}$ & -.00 & -.05 & $.25^{* *}$ & $-.09^{*}$ \\
\hline 6. Compras & 1.66 & .95 & & & & & & - & $.13^{\star *}$ & $.24^{* *}$ & $.19^{\star *}$ & $.33^{* *}$ & $.25^{\star *}$ & $.19^{* *}$ & $.21^{* *}$ & $.25^{* *}$ & $-.15^{\star *}$ & .081 & $-.19^{\star \star}-.17^{\star *}$ & .019 & .055 & $.16^{* *}$ & -.063 \\
\hline 7. Juegos & 3.11 & 1.30 & & & & & & & & $.27^{\star *}$ & .043 & $.36^{* *}$ & .07 & -.064 & .071 & .049 & -.07 & .037 & $-.066-.126^{*}$ & .028 & .07 & -.035 & .023 \\
\hline 8. Películas & 3.05 & 1.32 & 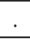 & & & & & & & - & $.28^{\star *}$ & $.40^{\star \star}$ & $.41^{\star \star}$ & .026 & $.27^{\star *}$ & $.35^{* *}$ & $-.21^{* \star}$ & .054 & $-.26^{\star *}-.23^{* *}$ & .028 &.-030 & $.17^{* *}$ & .018 \\
\hline 9. Fotografía & 3.64 & 1.14 & & . & & & & & & & - & $.47^{* *}$ & $.46^{\star *}$ & .036 & $.32^{* *}$ & $.38^{* *}$ & $-.23^{* *}$ & -.007 & $-.27^{* *}-.20^{* *}$ & -.068 & $-.09^{*}$ & $.29^{* *}$ & -.013 \\
\hline 10.Descargas & 3.16 & 1.21 & & & & & & & & & & - & $.45^{* *}$ & $.103^{*}$ & $.45^{* *}$ & $.41^{* \star}$ & $-.26^{* k}$ & $.112^{*}$ & $-.25^{* *}-.29^{* *}$ & -.031 & -.047 & $.22^{* *}$ & .035 \\
\hline 11. Música & 3.69 & 1.33 & & & & & & & & & & & - & .060 & $.48^{\star *}$ & $.50^{* *}$ & $-.30^{* *}$ & .039 & $-.37^{\star \star}-.21^{\star *}$ & -.010 & -.027 & $.32^{* *}$ & -.060 \\
\hline 12.Administración & 1.12 & 0.48 & & & & & & & & & & & & - & .082 & .028 & -.044 & -.002 & $-.101^{*}-.080$ & -.024 & .005 & $.103^{*}$ & -.055 \\
\hline 13. Frecuencia & 3.37 & 1.44 & & & . & & & & & & & & & & - & $.55^{\star \star}$ & $-.39^{* \star}$ & .072 & $-.34^{\star \star}-.30^{\star \star}$ & .002 & -.086 & $.34^{* *}$ & -.064 \\
\hline 14. Tiempo & 2.58 & 1.09 & & & . & & & & & & & & & & & - & $-.38^{* \star}$ & .095 & $-.43^{\star \star}-.34^{\star *}$ & .011 & -.029 & $.44^{* *}$ & -.072 \\
\hline 15. Beneficios & 7.63 & 2.71 & & & & & & & & & & & & & & & - & $.18^{* *}$ & $.33^{* *} .54^{* *}$ & .085 & $.24^{* *}$ & $-.33^{\star *}$ & .045 \\
\hline 16. Riesgos & 6.02 & 2.40 & & & & & & & & & & & & & & & & - & $-.13^{* *} .091$ & $.18^{\star *}$ & $.33^{* *}$ & -.042 & -.011 \\
\hline 17. Dificultad de control & 17.60 & 4.26 & & & & & & & & & & & & & & & & & $-.35^{* *}$ & $.25^{\star *}$ & .062 & $-.44^{* *}$ & $.119^{*}$ \\
\hline 18. Ventajas & 12.75 & 3.76 & & & & & & & & & & & & & & & & & - & $.21^{\star \star}$ & $.25^{* *}$ & $-.32^{\star \star}$ & .075 \\
\hline 19. Inconvenientes & 29.87 & 7.00 & & & & & & & & & & & & & & & & & & - & $.43^{* *}$ & $-.20^{* \star}$ & .069 \\
\hline 20. Efectos adversos & 17.09 & 5.50 & & & & & & & & & & & & & & & & & & & - & $-.12^{* \star}$ & -.063 \\
\hline 21.Uso problemático & 17.70 & 5.37 & & & . & & & & & & & & & & & & & & & & & - & $-.31^{* *}$ \\
\hline 22. Satisfacción vida & 19.02 & 4.63 & & & & & & & & & & & & & & & & & & & & & - \\
\hline
\end{tabular}

trol y, negativamente, pero en menor medida, con la frecuencia y el tiempo. El tiempo y la frecuencia se correlacionan entre sí y con los usos (ver Tabla 2).

\section{Análisis de varianza}

Comparando la percepción de riesgos y beneficios por parte de los padres y los menores, se observa que los beneficios percibidos en el uso del teléfono inteligente son superiores en los padres respecto a los menores ( $\mathrm{F}$ $=16.46 ; \mathrm{p}<.000)$, al igual que para los riesgos percibi$\operatorname{dos}(\mathrm{F}=5.15 ; \mathrm{p}<.024)$.
Respecto a la comparación en función del ciclo educativo (Tabla 3) se observan diferencias significativas en llamadas de voz, mensajería instantánea, redes sociales, consultar webs, fotografía, descargar aplicaciones, y escuchar música, con usos menores entre los estudiantes de primaria para todas ellas. También se observan diferencias en la frecuencia, duración y uso problemático del móvil, y son los estudiantes de bachillerato los que obtienen puntuaciones superiores. En cambio, para el uso de los juegos los estudiantes de primaria presentan puntuaciones más elevadas.

Tabla 3. Diferencias en usos, creencias, uso problemático y satisfacción vital según el ciclo educativo

\begin{tabular}{|c|c|c|c|c|c|}
\hline Nivel educativo & $\begin{array}{c}\text { Primaria } \\
\text { M (DT) }\end{array}$ & $\begin{array}{l}\text { Secundaria } \\
\text { M (DT) }\end{array}$ & $\begin{array}{c}\text { Bachillerato } \\
\text { M (DT) }\end{array}$ & $F$ & $\mathrm{p}$ \\
\hline Llamadas de voz & $2.31(1.12)$ & $3.09(1.07)$ & $3.47(1.16)$ & 23.72 & .000 \\
\hline Videoconferencias & $1.62(0.88)$ & $1.66(0.88)$ & $2.13(1.36)$ & 3.98 & .019 \\
\hline Mensajería instantánea & $2.56(1.42)$ & $4.59(0.81)$ & $4.81(0.47)$ & 171.55 & .000 \\
\hline Redes sociales & $2.51(1.50)$ & $3.82(1.28)$ & $4.06(1.10)$ & 40.70 & .000 \\
\hline Consulta web & $2.50(1.07)$ & $3.24(1.01)$ & $3.91(0.73)$ & 31.67 & .000 \\
\hline Compras en línea & $1.43(0.77)$ & $1.73(0.96)$ & $1.81(1.25)$ & 4.33 & .014 \\
\hline Juegos & $3.35(1.28)$ & $3.13(1.27)$ & 2.09 (1.08) & 12.27 & .000 \\
\hline Películas & $2.77(1.31)$ & $3.13(1.33)$ & $3.13(1.20)$ & 3.51 & .031 \\
\hline Fotografía & $3.20(1.16)$ & $3.76(1.09)$ & $4.13(1.00)$ & 13.27 & .000 \\
\hline Descargas & $2.69(1.21)$ & $3.29(1.13)$ & 3.59 (1.38) & 12.60 & .000 \\
\hline Música & $2.96(1.37)$ & $3.87(1.23)$ & $4.66(0.65)$ & 31.33 & .000 \\
\hline Administración & $1.06(0.35)$ & $1.11(0.41)$ & $1.38(1.00)$ & 5.68 & .004 \\
\hline Duración & $1.75(0.90)$ & $2.89(1.00)$ & $2.84(1.01)$ & 53.11 & .000 \\
\hline Frecuencia & $2.30(1.62)$ & $3.74(1.20)$ & $3.84(0.88)$ & 49.15 & .000 \\
\hline CERM & $14.45(4.07)$ & $18.69(5.27)$ & $20.38(5.43)$ & 32.90 & .000 \\
\hline Satisfacción & $20.01(3.91)$ & $18.82(4.69)$ & $17.84(5.67)$ & 4.92 & .008 \\
\hline Beneficios percibidos & $9.05(2.71)$ & $7.21(2.53)$ & $6.34(2.37)$ & 24.13 & .000 \\
\hline Dificultad de control & $19.30(4.11)$ & $17.00(4.17)$ & $16.91(4.06)$ & 12.48 & .000 \\
\hline Riesgos & $5.68(2.06)$ & $6.23(2.57)$ & $5.47(1.74)$ & 2.95 & .053 \\
\hline Ventajas & $14.21(3.92)$ & $12.29(3.58)$ & $11.75(3.37)$ & 12.03 & .000 \\
\hline Inconvenientes & $28.72(7.49)$ & $30.39(6.65)$ & $29.50(7.83)$ & 2.27 & .104 \\
\hline Efectos adversos & $17.27(5.50)$ & $16.93(5.47)$ & $17.91(5.88)$ & .52 & .591 \\
\hline
\end{tabular}


Respecto a las creencias, se observan diferencias en dificultad de control y ventajas, y las puntuaciones más elevadas las obtienen los estudiantes de primaria.

\section{Discusión}

Este estudio pretende conocer el uso que hacen los adolescentes del teléfono móvil inteligente, su percepción sobre los riesgos y beneficios, su relación con consecuencias negativas percibidas y su satisfacción con la vida. En lo relativo al uso del teléfono inteligente por parte de los adolescentes, el tiempo y la frecuencia de uso son similares a los de los adultos.

Respecto a las aplicaciones de este uso, las más frecuentes son las de mensajería instantánea y redes sociales, cámara de fotos, escuchar música, jugar a videojuegos y ver películas o videos, como en otros estudios (p. ej., Pontes et al., 2015), pero ligeramente diferente al uso que hacen los adultos, que es más frecuentemente en consultas web y en emisión o recepción de llamadas de voz. De forma consistente con las expectativas de los padres y los resultados de estudios previos (Carbonell et al., 2013) los menores utilizan poco el teléfono inteligente para tareas administrativas y compras, pero bastante para usos lúdicos. Sin embargo, los adolescentes muestran un uso más frecuente y mayor uso problemático del teléfono inteligente que los menores. Este resultado contrasta con la asunción de que el uso del teléfono móvil se normaliza con la edad y se le da un uso más práctico a medida que los adolescentes crecen (Moral \& Suárez, 2016), y es compatible con un incremento del uso problemático, posiblemente debido al aumento del uso derivado de la irrupción de las redes sociales en línea (Salehan \& Negahban, 2013).

En los resultados referentes al uso problemático, se observa que un $15 \%$ de los estudiantes se categorizan como población con un riesgo elevado. Estos datos coinciden con otros estudios (Gallimberti et al., 2016; Muñoz-Miralles et al., 2016) aunque algunas de las prevalencias se han obtenido a partir de instrumentos diferentes. El uso problemático del teléfono inteligente asociado con la mensajería instantánea, el uso de redes sociales y el uso lúdico o evasivo de escuchar música, así como con la alta frecuencia y el tiempo de uso, puede tener efectos perjudiciales en la salud. En este sentido, el uso problemático del teléfono inteligente puede ser considerado un indicador de riesgo (Adès \& Lejoyeux, 2003). Pero si ese uso problemático del teléfono inteligente derivara de una insatisfacción previa con la vida, interpretación que también se puede extraer de los resultados obtenidos en el presente estudio, esta conducta la podríamos explicar por la necesidad de búsqueda de contacto social y dependencia con el grupo de iguales para aliviar el malestar (Oberst, Wegmann, Stodt, Brand \& Chamarro, 2017).

Respecto a la satisfacción vital, los resultados confirman que los adolescentes que usan el teléfono inteligente de forma abusiva están menos satisfechos con la vida, aunque podría ser que aquellos estudiantes con una baja satisfacción con la vida son los que utilizan el teléfono inteligente de forma problemática. En todo caso, el 69.9\% de los adolescentes están satisfechos con su vida de forma similar a otros estudios realizados anteriormente en el contexto español (Chavarría \& Barra, 2014) y global (Proctor, Linley, \& Maltby, 2009). Según estos autores, la mayoría de los estudios muestran que tanto los infantes como los adolescentes refieren altos niveles de satisfacción vital.

En relación con el nivel educativo, se observa que el uso problemático del teléfono inteligente es mayor en secundaria y bachillerato. Estos resultados podrían explicarse por la frecuencia y duración del uso. Por otra parte, se observa un uso de las funciones del teléfono inteligente distinto en cada etapa, y son las redes sociales, la consulta de webs, fotos, descargas, música y mensajería instantánea las que más se utilizan por parte de los adolescentes mayores. En cambio, por parte de los estudiantes de primaria su interés principal son los juegos. También hay diferencias relativas a las creencias de los estudiantes sobre el uso del teléfono inteligente. Los estudiantes de primaria perciben mayores beneficios en el uso, pero también una mayor dificultad de control. Por tanto, estos resultados sugieren que un alumno de primaria no percibe de la misma manera los riesgos y beneficios que los alumnos más mayores. La explicación podría venir del hecho de que los adolescentes han estado más expuestos a este dispositivo, conocen todas sus funciones y están más alertados de sus posibles ventajas e inconvenientes. Estos datos son interesantes porque permiten plantear la importancia de potenciar el uso saludable del teléfono inteligente en etapas más tempranas, donde la presencia del mismo tiene una incidencia considerable. Por ello, creemos necesario hacerlo antes de la secundaria, ya que la aparición de este dispositivo se da en ciclos inferiores, donde los niños tienen menos conocimientos y consciencia del posible uso indebido y de los riesgos del teléfono inteligente que tienen o tendrán en un futuro.

\section{Discusión general}

Nuestro trabajo tenía por objetivo conocer: a) los usos que hacen los adultos del móvil y su percepción de los riesgos y beneficios que comporta el uso del móvil por parte de sus hijos, y b) el uso que hacen los adolescentes, su percepción sobre los riesgos y beneficios, y su relación con consecuencias negativas percibidas y su satisfacción con la vida. Respecto al primero, los resultados que indican los usos principales como comunicativos, recreativos y prácticos, sugieren que, en el momento actual, el uso práctico y con potenciales beneficios parece destacar. También hemos observado que el tiempo y la frecuencia de uso por parte de los adultos y los menores tienden a equipararse, lo que sugiere un incremento progresivo del uso en los adolescentes, aunque se descarta un uso excesivo en los menores. En todo caso, los adultos creen que los menores utilizan el 
móvil menos de lo que lo hacen realmente, por lo que es razonable pensar que las posibilidades que tienen de detectar un uso problemático en sus hijos se vean disminuidas. Respecto a los tipos de usos, se aprecian similitudes y diferencias. Como similitudes, adultos y adolescentes utilizan con gran frecuencia la mensajería instantánea, las redes sociales y la cámara de fotos. Como diferencias, los adolescentes utilizan más el móvil para jugar, acceder a contenido multimedia, escuchar música y descargar aplicaciones en Internet, mientras que los adultos lo utilizan más para acceder a Internet y hacer consultas. Estos resultados son consistentes con la idea de que los adolescentes y los menores, a pesar de que la edad de acceso al uso de los móviles disminuye, han aprendido a integrar la tecnología en sus vidas (Martínez de Morentín et al., 2014). En todo caso, como en estudios anteriores (Malo et al., 2012) destaca el uso de tipo comunicativo complementado con otros usos lúdicos (Eynon \& Malmberg, 2011). Parece que los menores están evolucionando del uso principalmente lúdico (Carbonell et al., 2013) hacia el uso comunicativo, posiblemente facilitado por la eclosión de las redes sociales (Salehan \& Negahban, 2013), lo que indica, de acuerdo con Carbonell \& Panova (2017) que utilizan la tecnología de una forma positiva. Estos resultados contrastan con la percepción de los padres respecto de los usos del móvil por parte de los hijos, ya que se limita al uso lúdico y obvian el uso comunicativo potenciado por las redes sociales. Es importante destacar que actualmente se considera red social a cualquier sitio web que permita la interacción social, por lo que mundos virtuales de juego o canales de YouTube, ampliamente utilizados por los menores, pueden ser espacios de intercambio (McBride, 2011). Ante esta discrepancia, creemos que los padres han de entender que usar el teléfono móvil es una nueva forma de emplear el tiempo libre para sus hijos (Plaza de la Hoz, 2017) y que esta nueva actividad necesariamente incluirá la interacción en las redes sociales.

Respecto a las creencias de los padres, parece que el uso del móvil es tanto una fuente de riesgos como de beneficios, al menos más claramente que los menores. Y en relación con el menor, no perciben el uso del teléfono móvil como la oportunidad de crecimiento personal y el desarrollo de capacidades.

Esta actitud, que catalogaríamos de "prudente" creemos que puede situar a los padres en una buena posición como educadores del uso del móvil de sus hijos, siempre y cuando estén verdaderamente capacitados para ello. Como señalan O'Keeffe et al. (2011), muchos adultos usan la tecnología increíblemente bien y se sienten capaces de supervisar los lugares que sus hijos visitan, aunque a menudo hay padres que no comprenden que la actividad en línea de sus hijos es una extensión de su vida real. Por todo ello quizá los padres tengan mucho que aprender sobre el comportamiento de sus hijos en línea (McBride, 2011), así como sobre las recomendaciones de respetar restricciones en sitios web y aplicaciones, pues permitir que los niños falsifiquen su edad les pone en riesgo de ver contenidos inapropiados además de enviar un mensaje confuso sobre la seguridad en Internet y la mentira. La autoridad de los mayores, además, queda cuestionada, y entra en conflicto con las nuevas formas de ser informado (Plaza de la Hoz, 2017). Los adolescentes están descubriendo que mediante Internet pueden resolver cuestiones relacionadas con sus preocupaciones, sin embargo, pueden no interpretar correctamente esa información. En todo caso, no considerar el uso del móvil como una herramienta con potencial para que el menor se comunique y explore usos beneficiosos puede suponer un freno al uso adaptativo (O'Keeffe et al., 2011). Si este aprendizaje no lo hacen con la ayuda de sus padres será una tarea que deberán aprender y practicar en otro entorno, normalmente de la mano de otros menores o adolescentes, lo que sin duda implica un riesgo elevado de aprendizaje de usos no adecuados a la edad.

Finalmente, hay que considerar las limitaciones del presente estudio, centradas especialmente en el muestreo intencional y su diseño transversal. Futuros estudios podrían estudiar la relación entre las actitudes y los comportamientos de los padres y sus propios hijos, tanto de forma transversal como longitudinal.

\section{Implicaciones y conclusiones}

Tal y como hemos podido observar en los resultados de estas investigaciones, el uso del teléfono móvil es diverso y deben tenerse en cuenta múltiples aspectos antes de determinar si se está o no haciendo un uso problemático del mismo. Los menores y adolescentes buscan descubrir, explorar e investigar las potencialidades de estos dispositivos, aunque hacerlo por sí mismos, sin ningún tipo de aprendizaje previo, puede conducirlos a experimentar situaciones inadecuadas para la edad. La falta de madurez y las habilidades para gestionar contenidos adultos puede derivar en actitudes y conductas inapropiadas e incluso conducir a algunos de ellos a experimentar malestar psicológico. Indudablemente, los adultos responsables de estos menores tienen un papel clave en este momento del desarrollo y en su educación. Por ello es necesario que tomen conciencia de las implicaciones del uso del teléfono móvil, tanto positivas como negativas. Aún así, ocurre que valorar bien los riesgos no es tarea sencilla sobre todo cuando no existe consenso sobre cuáles son exactamente esos riesgos. Por ello consideramos necesario explorar aún más, en futuras investigaciones, los usos y sus consecuencias en la vida psíquica de los menores, ya que, aunque los adultos dicen reconocer los efectos negativos del uso en menores, la conducta permisiva persiste. La falta de conciencia en los adultos sobre los efectos del uso del teléfono móvil en menores, que puede estar relacionada con la desajustada percepción de los riesgos y con la creencia errónea sobre el tipo de uso que sus hijos hacen de esta tecnología, nos obliga a considerar el fenómeno de la expansión del teléfono móvil entre los más pequeños como un factor de riesgo en el de- 
sarrollo de actitudes y conductas relacionadas con el uso problemático de estos dispositivos. Por ello consideramos también necesario valorar el desarrollo de recursos orientados a capacitar y empoderar a los adultos para enseñar a los menores a manejar con autonomía y responsabilidad las nuevas tecnologías.

\section{Referencias}

Adès, J. \& Lejoyeux, M. (2003). Las nuevas adicciones Internet, sexo, juego, deporte compras trabajo dinero. Barcelona: Kairós.

Atienza, F., Pons, D., Balaguer, I., \& García-Merita, M. (2000). Propiedades psicométricas de la Escala de Satisfacción con la Vida en adolescentes. Psicothema, 12(1), 314-319.

Beranuy, M., Chamarro, A., Graner, C., \& Carbonell, X., (2009). Validación de dos escalas breves para evaluar la adicción a Internet y el abuso de móvil. Psicothema, 21, 480-485.

Bringué, X. \& Sábada, Ch. (2011). La Generación Interactiva en Madrid. Niños y adolescentes ante las pantallas. Foro Generaciones Interactivas - Fundación Telefónica. Recuperado el 5 de marzo de http:// dadun.unav.edu/bitstream/10171/20593/1/GGIIMadrid-final.pdf

Carvalho, J., Fonseca, G., Francisco, R., Bacigalupe, G., \& Relvas, A. (2016). Information and communication technologies and family: Patterns of use, life cycle and family dinamics. Journal of Psychology \& Psychotherapy, 6, 10-12. https://doi.org/10.4172/ 2161-0487.1000240

Carbonell, X., Fuster, H., Chamarro, A., \& Oberst, U. (2012). Adicción a Internet y móvil: una revisión de estudios empíricos españoles. Papeles del psicólogo, 33(2), 82-89.

Carbonell, X., Oberst, U., \& Beranuy, M. (2013). The cell phone in the Twenty-First Century: A risk for addiction or a necessary tool? Principles of Addiction, 1, 901-909.

Carbonell, X. \& Panova, T. (2017). A critical consideration of social networking sites' addiction potential. Addiction Research \& Theory, 25, 48-57. https://doi.or g/10.1080/16066359.2016.1197915

Chavarría, M. P. \& Barra, E. (2014). Satisfacción Vital en Adolescentes: Relación con la Autoeficacia y el Apoyo Social Percibido. Terapia Psicológica, 32, 41-46. https://doi.org/10.4067/S0718-48082014000100004

Diener, E., Emmons, R., Larsen, R., \& Griffin, S. (1985). The Satisfaction with Life Scale. Journal of Personality Assessment, 49, 71-75. https://doi.org/10.1207/ s15327752jpa4901_13

Ditrendia (2016). Informe Mobile en España y en el Mundo 2016. Recuperado el 5 de marzo de http://www. ditrendia.es/informe-ditrendia-mobile-en-espana-yen-el-mundo-2016/

Eynon, R., \& Malmberg, L. E. (2011). A typology of young people's Internet use: Implications for education. Computers \& Education, 56, 585-595. https:// doi.org/10.1016/j.compedu.2010.09.020
Fundación ANAR (2016). I Estudio sobre ciberbullying según los afectados. Recuperado el 28 de octubre de https://www.anar.org/wp-content/uploads/2017/03/ INFORME-I-ESTUDIO-BULLYING.pdf

Gallimberti, L., Buja, A., Chindamo, S., Terraneo, A., Marini, E., Rabensteiner, A., ... Baldo, V. (2016). Problematic cell phone use for text messaging and substance abuse in early adolescence (11- to 13-yearolds). European Journal of Pediatrics, 175(3), 355-364. https://doi.org/10.1007/s00431-015-2645-y

Instituto Nacional de Estadística (2016). Encuesta sobre Equipamiento y Uso de Tecnologías de la Información y la Comunicación en los Hogares, 2016. Recuperado el 5 de marzo de http://www.ine.es/prensa/ np991.pdf

Instituto Nacional de Tecnologías de la Comunicación (2010). Estudio sobre seguridad y privacidad en el uso de los servicios móviles por los menores españoles. Recuperado el 5 de marzo de http://acercadeorange.orange. es/UpImages/files/2204/estudio_intecoorange_ menores_y_moviles_31e23c99b05123c622f224dfa.pdf

Labrador, F. J. \& Villadangos, S. M. (2010). Menores y nuevas tecnologías: conductas indicadoras de posible problema de adicción. Psicothema, 22(2), 180-188,

Malo, S., Navarro, D., \& Casas, F. (2012). El uso de los medios audiovisuales en la adolescencia y su relación con el bienestar subjetivo: análisis cualitativo desde la perspectiva intergeneracional y de género. Athenea Digital: Revista de Pensamiento e Investigación Social, 12, 27-49.

Martínez de Morentín, J. I., Cortés, A., Medrano, C., \& Apodaca, P. (2014). Internet use and parental mediation: A cross-cultural study. Computers \& Education, 70, 212-221. https://doi.org/10.1016/j.compedu.2013.07.036

McBride, D. L. (2011). Risks and benefits of social media for children and adolescents. Journal of Pediatric Nursing, 26, 498-499. https://doi.org/10.1016/j. pedn.2011.05.001

Moral, M. de la V. \& Suárez, C. (2016). Factores de riesgo en el uso problemático de Internet y del teléfono móvil en adolescentes españoles. Revista Iberoamericana de Psicología y Salud, 7(2), 69-78. https:// doi.org/10.1016/j.rips.2016.03.001

Muñoz-Miralles, R., Ortega-González, R., Batalla-Martínez, C., López-Morón, M. R., Manresa, J. M., \& Torán-Monserrat, P. (2014). Acceso y uso de nuevas tecnologías entre los jóvenes de educación secundaria, implicaciones en salud. Estudio JOITIC. Atención Primaria, 46, 77-88. https://doi.org/10.1016/j. aprim.2013.06.001

Muñoz-Miralles, R., Ortega-González, R., López-Morón, M. R., Batalla-Martínez, C., Manresa, J. M., MontellàJordana, N., ... Torán-Monserrat, P. (2016). The problematic use of Information and Communication Technologies (ICT) in adolescents by the cross sectional JOITIC study. BMC Pediatrics, 16, 140. https:// doi.org/10.1186/s12887-016-0674-y

Oberst, U., Wegmann, E., Stodt, B., Brand, M., \& Chamarro, A. (2017). Negative consequences from heavy 
social networking in adolescents: The mediating role of fear of missing out. Journal of Adolescence, 55, 5160. https://doi.org/10.1016/j.adolescence.2016.12.008

O'Keeffe, G. S., Clarke-Pearson, K., \& Council on Communications and Media (2011). The impact of Social Media on children, adolescents, and families. PEDIATRICS, 127, 800-804. https://doi.org/10.1542/ peds.2011-0054

Padrós, F., Gutiérrez, C. Y., \& Medina, M. A. (2015). Propiedades Psicométricas de la escala de satisfacción con la vida (SWLS) de Diener en población de Michoacán (México). Avances en Psicología Latinoamericana, 33, 223-232. https://doi.org/10.12804/ apl33.02.2015.04

Plaza de la Hoz, J. (2017). Advantages and downsides of children ICT use in Spain: The parent's perspective. International Journal on Lifelong Education and Leadership, 3, 22-31.

Pontes, H. M., Szabo, A., \& Griffiths, M. D. (2015). The impact of Internet-based specific activities on the perceptions of Internet addiction, quality of life, and excessive usage: A cross-sectional study. Addictive Behaviors Reports, 1, 19-25. https://doi.org/10.1016/j. abrep.2015.03.002

Proctor, C., Linley, P., \& Maltby, J. (2009). Youth life satisfaction: A review of the literature. Journal of Happiness Studies, 10, 583-630. https://dx.doi. org/10.1007/s10902-008-9110-9

Rial, A., Gómez, P., Varela, J., \& Braña, T. (2014). Actitudes, percepciones y uso de internet y las redes sociales entre los adolescentes de la comunidad gallega. Anales de Psicología, 30, 642-655. https://doi. org/10.6018/analesps.30.2.159111
Romero-Ruiz, K., Echeverri-Sánchez, L., Peña-Plata, J., Vásquez-Giraldo, S., Aguilera-Cardona, M., HerazoAvendaño, C., ... Bran-Piedrahita, L. (2017). Information and Communication Technologies Impact on Family Relationship. Global Journal of Health Science, 237, 30-37. https://doi.org/10.1016/j.sbspro.2017.02.007

Ruiz, J., Sánchez, J., \& Trujillo, J. (2016). Utilización de Internet y dependencia a teléfonos móviles en adolescentes. Revista Latinoamericana de Ciencias Sociales, Niñez y Juventud, 14, 1357-1369.

Salehan, M., \& Negahban, A. (2013). Social networking on smartphones: When mobile phones become addictive. Computers in Human Behavior, 29(6), 26322639. https://doi.org/10.1016/j.chb.2013.07.003

Sánchez-Carbonell, X., Beranuy, M., Castellana, M., Chamarro, A., \& Oberst, U. (2008). La adicción a Internet y al móvil: ¿moda o trastorno? Adicciones, 20(2), 149-160.

Valencia, A., Sánchez González, A., Garcés Bedoya, J. C., \& González Saldarriaga, M. A. (2015) Vínculos en red: reflexiones desde la presencialidad. Revista Poiésis, 30, 131-137.

Valkenburg, P. M., Peter, J., \& Schouten, A. P. (2006). Friend networking sites and their relationship to adolescents' well-being and social self-esteem. $C y$ berPsychology \& Behavior, 9(5), 584-590. https://doi. org/10.1089/cpb.2006.9.584

Valkenburg, P. M., \& Peter, J. (2009). Social consequences of the Internet for adolescents. A decade of research. Current Directions in Psychological Science, 18, 1-5. https:// doi.org/10.1111/j.1467-8721.2009.01595.x 
